\title{
Torsion in free center-by-nilpotent-by-abelian Lie rings of rank 2
}

DOI:

10.1080/00927872.2017.1350699

\section{Document Version}

Accepted author manuscript

Link to publication record in Manchester Research Explorer

\section{Citation for published version (APA):}

Stöhr, R. (2017). Torsion in free center-by-nilpotent-by-abelian Lie rings of rank 2. Communications in Algebra, 18. https://doi.org/10.1080/00927872.2017.1350699

\section{Published in:}

Communications in Algebra

\section{Citing this paper}

Please note that where the full-text provided on Manchester Research Explorer is the Author Accepted Manuscript or Proof version this may differ from the final Published version. If citing, it is advised that you check and use the publisher's definitive version.

\section{General rights}

Copyright and moral rights for the publications made accessible in the Research Explorer are retained by the authors and/or other copyright owners and it is a condition of accessing publications that users recognise and abide by the legal requirements associated with these rights.

\section{Takedown policy}

If you believe that this document breaches copyright please refer to the University of Manchester's Takedown Procedures [http://man.ac.uk/04Y6Bo] or contact uml.scholarlycommunications@manchester.ac.uk providing relevant details, so we can investigate your claim.

\section{OPEN ACCESS}




\title{
TORSION IN FREE CENTRE-BY-NILPOTENT-BY-ABELIAN LIE RINGS OF RANK 2
}

\author{
RALPH STÖHR
}

\begin{abstract}
For $c \geq 2$, the free centre-by-(nilpotent-of-class-c-1)-by abelian Lie ring on a set $X$ is the quotient $L /\left[\left(L^{\prime}\right)^{c}, L\right]$ where $L$ is the free Lie ring on $X$, and $\left(L^{\prime}\right)^{c}$ denotes the $c$ th term of the lower central series of the derived ideal $L^{\prime}=L^{2}$ of $L$. In this paper we give a complete description of the torsion subgroup of its additive group in the case where $|X|=2$ and $c$ is a prime number.
\end{abstract}

\section{INTRODUCTION}

For an integer $c \geq 2$, the free centre-by-(nilpotent-of-class $c-1$ )-by-abelian Lie ring on a set $X$ is the quotient

$$
L /\left[\left(L^{\prime}\right)^{c}, L\right]
$$

where $L$ is an (absolutely) free Lie ring on $X$ and $\left(L^{\prime}\right)^{c}$ is the $c$ th term of the lower central series of the derived ideal $L^{\prime}=L^{2}$ of $L$. In this note we determine the torsion subgroup of the additive group of (1.1) in the case where $L$ has rank 2 , that is $X$ is a set of two elements, and $c$ is a prime number. It is easily observed that for any $c \geq 2$ and any $X$ with $|X| \geq 2$ the torsion subgroup of (1.1) is contained in the central ideal $\left(L^{\prime}\right)^{c} /\left[\left(L^{\prime}\right)^{c}, L\right]$. If $c=p$, where $p$ is a prime, then the ideal $\left(L^{\prime}\right)^{p} /\left[\left(L^{\prime}\right)^{p}, L\right]$ is a direct sum of a free abelian group and an infinite elementary abelian $p$-group. We exhibit an explicit $\mathbb{Z}_{p}$-basis of the torsion part if $|X|=2$. This is the main result of this paper, see Theorem 5.1 in Section 5. In the earlier paper [1] a complete description of the torsion subgroup of the additive group of the more reduced quotient

$$
L /\left(\left[\left(L^{\prime}\right)^{c}, L\right]+L^{\prime \prime \prime}\right)
$$

was obtained, again in the case of rank 2 , but for arbitrary $c \geq 2$. Note that (1.2) coincides with (1.1) for $c=2$ and $c=3$, but not for $c \geq 4$. We make essential use of the results from [1].

The interest in torsion in relatively free Lie rings of the form (1.1) as well as their group-theoretic counterparts, the relatively free groups $F /\left[\gamma_{c}\left(F^{\prime}\right), F\right]$, where $F$ is a free group and $\gamma_{c}\left(F^{\prime}\right)$ is the $c$ th term of the lower central series of the derived subgroup $F^{\prime}$, has a long history. If $c=2$, these turn into the free centre-bymetabelian Lie rings and the free centre-by-metabelian groups, respectively, and it was the latter in which Kanta Gupta [8] first discovered torsion elements, a major

2010 Mathematics Subject Classification. Primary 17B01, 17 B55.

Key words and phrases. Free Lie rings. 
surprise at the time. Later torsion was detected in $F /\left[\gamma_{c}\left(F^{\prime}\right), F\right]$ if $c$ is a prime [15] and if $c=4$ [16]. Quite surprising, though, it turned out that $F /\left[\gamma_{c}\left(F^{\prime}\right), F\right]$ is torsion free if $c$ is divisible two distint primes [10]. As to Lie rings, early work in [12] on the free centre-by-metabelian Lie rings $L /\left[L^{\prime \prime}, L\right]$, and in particular on torsion in the additive group, turned out in need of some rectification, and this was eventually accomplished in [14] and [11]. For larger values of $c$, Drensky [7] proved that for any prime $p$ the free Lie ring $L /\left[\left(L^{\prime}\right)^{p}, L\right]$ contains non-trivial multilinear elements of degree $2 p+1$ which have order $p$.

The paper is organized as follows. In Section 2 we introduce notation and assemble a number of preliminary results on the homogeneous components of free Lie rings. These are further examined in Section 3 where we derive our main result on Lie powers of free modules in prime degree, postponing, however, one key ingredient on Lie powers of prime degree $p$ over fields of characteristic $p$ to Section 4 . In the final Section 5 we exploit the results of the previous sections to derive our main result.

\section{Notation AND SOME PRELIMINARIES}

We write maps on the right and use left-normed notation for Lie products. Let $A$ be a free abelian group. By $L(A)$ we denote the free Lie ring on $A$. For a positive integer $c$, we let $L^{c}(A)$ denote the degree $c$ homogeneous component of $L(A)$, that is the span of all left normed simple Lie products $\left[a_{1}, a_{2}, \ldots, a_{c}\right]$ with $a_{i} \in A$. In particular, $L^{1}(A)=A$. The universal envelope of $L(A)$ can be identified with the tensor $\operatorname{ring} T(A)=\bigoplus_{c \geq 0} T^{i}(A)$ where $T^{0}(A)=\mathbb{Z}$ and, for $c>0, T^{c}(A)=\underbrace{A \otimes \cdots \otimes A}_{c}$, the $c$ th tensor power of $A$. By $A^{c}$ we denote the $c$ th symmetric power of $\stackrel{c}{A}$. The free metabelian Lie ring on $A$ is the quotient of $L(A)$ by its second derived ideal: $M(A)=L(A) / L(A)^{\prime \prime}$. This too is a graded Lie ring and we let $M^{c}(A)$ denote its cth homogeneous component, that is $M^{c}(A)=L^{c}(A) /\left(L^{c}(A) \cap L(A)^{\prime \prime}\right)$. We call $L^{c}(A)$ and $M^{c}(A)$ the $c$ th free Lie power and the $c$ th free metabelian Lie power of $A$, respectively. It is well-known that if $\mathcal{A}$ is an ordered $\mathbb{Z}$-basis of $A$, then the left normed simple Lie products $\left[b_{1}, b_{2}, \ldots, b_{c}\right]$ with $b_{i} \in \mathcal{A}$ and $b_{1}>b_{2} \leq \cdots \leq b_{c}$ form a $\mathbb{Z}$-basis of $M^{c}(A)$ (see [2, Section 4.2.2]).

The canonical embedding of $L(A)$ into its universal envelope $T(A)$ induces in each degree $c$ an embedding $\nu_{c}: L^{c}(A) \rightarrow T^{c}(A)$. By a well-known theorem of Wever (see [13, Chapter 5, Theorem 5.16]), the composite of this embedding with the natural projection $\rho_{c}: T^{c}(A) \rightarrow L^{c}(A)$ defined by $a_{1} \otimes \cdots \otimes a_{c} \mapsto\left[a_{1}, \ldots, a_{c}\right]$ amounts to multiplication by $c$ on $L^{c}(A)$ :

$$
L^{c}(A) \stackrel{\nu_{c}}{\longrightarrow} T^{c}(A) \stackrel{\rho_{c}}{\longrightarrow} L^{c}(A), \quad \nu_{c} \rho_{c}=c .
$$

The definition of $M^{c}(A)$ gives rise to a short exact sequence

$$
0 \rightarrow B^{c}(A) \rightarrow L^{c}(A) \stackrel{\eta_{c}}{\longrightarrow} M^{c}(A) \mapsto 0
$$


where $B^{c}(A)=L^{c}(A) \cap L(A)^{\prime \prime}$ and $\eta_{c}$ is the natural projection map. Moreover, for $c \geqslant 2$ the metabelian Lie power $M^{c}(A)$ fits into a short exact sequence

$$
0 \rightarrow M^{c}(A) \stackrel{\mu_{c}}{\longrightarrow} A \otimes A^{c-1} \stackrel{\kappa_{c}}{\longrightarrow} A^{c} \rightarrow 0
$$

where the maps $\mu_{c}$ and $\kappa_{c}$ are given by

$$
\left[a_{1}, a_{2}, \ldots, a_{c}\right] \mapsto a_{1} \otimes\left(a_{2} \circ \cdots \circ a_{c}\right)-a_{2} \otimes\left(a_{1} \circ \cdots \circ a_{c}\right)
$$

and $a_{1} \otimes\left(a_{2} \circ \cdots \circ a_{c}\right) \mapsto a_{1} \circ a_{2} \circ \cdots \circ a_{c}$, respectively (see [9, Corollary 3.2]). Moreover, there is a map $\lambda_{c}: A \otimes A^{c-1} \rightarrow M^{c}(A)$, given by

$a_{1} \otimes\left(a_{2} \circ \cdots \circ a_{c}\right) \mapsto\left[a_{1}, a_{2}, a_{3}, \ldots, a_{c}\right]+\left[a_{1}, a_{3}, a_{2}, \ldots, a_{c}\right]+\cdots+\left[a_{1}, a_{c}, a_{2}, \ldots, a_{c-1}\right]$,

such that the composite of $\mu_{c}$ and $\lambda_{c}$ amounts to multiplication by $c$ on $M^{c}(A)$ :

$$
M^{c}(A) \stackrel{\mu_{c}}{\longrightarrow} A \otimes A^{c-1} \stackrel{\lambda_{c}}{\longrightarrow} M^{c}(A), \quad \quad \mu_{c} \lambda_{c}=c
$$

see [1, Section 3]. Finally, for $c \geq 2$ there is a map $\theta_{c}: M^{c}(A) \rightarrow L^{c}(A)$ given by

$$
\left[a_{1}, \ldots, a_{c}\right] \mapsto \frac{1}{c}\left(\sum_{\sigma}\left[a_{1}, a_{\pi(2)}, \ldots, a_{\pi(c)}\right]-\sum_{\tau}\left[a_{2}, a_{\tau(1)}, \ldots, a_{\tau(c)}\right]\right)
$$

where the sums run over all permutations $\sigma$ of $\{2,3, \ldots, n\}$ and all permutations $\tau$ of $\{1,3, \ldots, n\}$, respectively. Although we work over $\mathbb{Z}$, the factor $1 / c$ in $(2.5)$ makes sense as the expression on the right hand side can be written as a Lie polynomial with integer coefficients(see [4, Section 2]). This Lie polynomial has been calculated in [5, Proposition 7.3], but since it is rather involved, we prefer to use the compact form (2.5) in what follows. The composite of $\theta_{c}$ and the natural projection $\eta_{c}$ as in (2.2) amounts to multiplication by $(c-2)$ ! on $M^{c}(A)$ :

$$
M^{c}(A) \stackrel{\theta_{c}}{\longrightarrow} L^{c}(A) \stackrel{\eta_{c}}{\longrightarrow} M^{c}(A), \quad \theta_{c} \eta_{c}=(c-2) !
$$

(see [4, Section 2]).

Now suppose that $A$ carries the structure of a module for the polynomial ring $U=\mathbb{Z}[X]$ where $X$ is a finite set of variables. Then all the objects introduced in this section such as Lie powers, symmetric powers etc. will be regarded as $U$-modules under the derivation action. For example, for $x \in X, a_{i} \in A$,

$$
\left[a_{1}, a_{2}, \ldots, a_{c}\right] x=\sum_{i=1}^{c}\left[a_{1}, \ldots, a_{i} x, \ldots, a_{c}\right]
$$

Note that all the maps introduced in this section are compatible with the derivation action, that is, all these maps are, in fact, $\mathbb{Z}[X]$-module homomorphisms. This will be used in what follows without further reference being given. The ring of integers $\mathbb{Z}$ will be regarded as a trivial $U$-module.

\section{LIE POWERS OF FREE MODULES}

In this section we retain the notation introduced in Section 2, but now we assume throughout that $A$ is a free $U$-module for the polynomial ring $U=\mathbb{Z}(X)$. All homology groups in this section will be over the ground ring $U$. For brevity, if $W$ is a $U$-module, the homology groups $H_{k}(U, W)=\operatorname{Tor}_{k}^{U}(W, \mathbb{Z}), k \geq 0$, will be written as $H_{k}(W)$. 
It is well known (see, for example, [14, Lemma 5.2]) that if $A$ is a free $U$-module then both $T^{c}(A)$ and $A \otimes A^{c-1}$ with $c \geq 2$ are also free $U$-modules under the derivation action.

Lemma 3.1. Let $A$ be a free $U$-module, $c \geq 2$. Then

(i) the tensor products $L^{c}(A) \otimes_{U} \mathbb{Z}$ and $M^{c}(A) \otimes_{U} \mathbb{Z}$ are direct sums of a free abelian group and a torsion group of exponent dividing $c$,

(ii) for $k \geq 1$ the homology groups $H_{k}\left(L^{c}(A)\right)$ and $H_{k}\left(M^{c}(A)\right)$ are torsion groups of exponent dividing $c$.

Proof. By applying the homology functor to the maps in (2.1) we get that $H_{k}\left(\nu_{c} \rho_{c}\right)$ is multiplication by $c$ on $H_{k}\left(L^{c}(A)\right)$ for all $k \geq 0$. Since $A$ is a free $U$-module, $H_{0}\left(T^{c}(A)\right)$ is free abelian and $H_{k}\left(T^{c}(A)\right)=0$ for $k \geq 1$. Then, for any $u \in H_{k}\left(L^{c} A\right)$ with $k>0$,

$$
c u=u H_{k}\left(\nu_{c} \rho_{c}\right)=\left(u H_{k}\left(\nu_{c}\right)\right) H_{k}\left(\rho_{c}\right)=0 H_{k}\left(\rho_{c}\right)=0 .
$$

The same holds if $k=0$ and $u \in \operatorname{Ker}\left(H_{0}\left(\nu_{c}\right)\right)$. Hence multiplication by $c$ annihilates both the homology groups $H_{k}\left(L^{c}(A)\right)$ for $k \geq 1$ and the kernel of the homomorphism $H_{0}\left(\nu_{c}\right)$. The image of this homomorphism is contained in the free abelian group $H_{0}\left(T^{c}(A)\right.$ ), and hence itself free abelian. The results (i) and (ii) for $L^{c}(A)$ follow. The proof of (i) and (ii) for $M^{c}(A)$ are obtained by a similar argument using the maps in (2.4) instead of those in (2.1).

Now we consider Lie powers of prime degree. Let $p$ be a prime. By applying the homology functor to the short exact sequence (2.2) we obtain the exact sequence $(3.1) \cdots \rightarrow H_{1}\left(M^{p}(A)\right) \rightarrow B^{p}(A) \otimes_{U} \mathbb{Z} \rightarrow L^{p}(A) \otimes_{U} \mathbb{Z} \stackrel{\eta_{p} \otimes 1}{\longrightarrow} M^{p}(A) \otimes_{U} \mathbb{Z} \mapsto 0$.

By Lemma 3.1., $L^{p}(A) \otimes_{U} \mathbb{Z}$ is a direct sum of a free abelian group and an elementary abelian $p$-group, and $H_{1}\left(M^{p}(A)\right)$ is an elementary abelian $p$-group. Note that this does not exclude the possibility that these torsion subgroups are trivial. Now the exact sequence (3.1) yields that $B^{p}(A) \otimes_{U} \mathbb{Z}$ is a direct sum of a free abelian group and a (possibly trivial) $p$-group of exponent dividing $p^{2}$. In fact, we will show that this group has actually no torsion, in other words, we will prove the following result.

Lemma 3.2. Let $A$ be a free $U$-module and $p$ a prime. Then the tensor product $B^{p}(A) \otimes_{U} \mathbb{Z}$ is a free abelian group.

Proof. Since we already know that $B^{p}(A) \otimes_{U} \mathbb{Z}$ is a direct sum of a free abelian group and a $p$-group of finite exponent, it is sufficient to show that no non-zero element in $B^{p}(A) \otimes_{U} \mathbb{Z}$ is annihilated by $p$. We use reduction modulo $p$, that is the short exact sequence

$$
0 \rightarrow B^{p}(A) \stackrel{p}{\rightarrow} B^{p}(A) \rightarrow B^{p}(A) \otimes \mathbb{Z}_{p} \rightarrow 0
$$

which, in its turn, gives rise to the exact sequence

$(3.2) \cdots \rightarrow H_{1}\left(B^{p}(A) \otimes \mathbb{Z}_{p}\right) \rightarrow B^{p}(A) \otimes_{U} \mathbb{Z} \stackrel{p}{\rightarrow} B^{p}(A) \otimes_{U} \mathbb{Z} \rightarrow B^{p}(A) \otimes_{U} \mathbb{Z}_{p} \rightarrow 0$.

The Lemma will be proved once we show that the homology group on the left is zero, and this will certainly follow if we can verify that $B^{p}(A) \otimes \mathbb{Z}_{p}$, regarded as a 
module for the polynomial ring $\mathbb{Z}_{p}[X]$, is projective. The proof of this fact will be given in the next section (see Corollary 4.2). This will then complete the proof of Lemma 3.2.

Now we have all the ingredients in place to prove the main result of this section. Recall the homomorphism $\theta_{c}: M^{c}(A) \rightarrow L^{c}(A)$ defined by (2.5).

Proposition 3.3. Let $A$ be a free $U$-module and $p$ a prime. Then the torsion subgroups of $L^{p}(A) \otimes_{U} \mathbb{Z}$ and $M^{p}(A) \otimes_{U} \mathbb{Z}$ are isomorphic, and the homomorphism $\theta_{p} \otimes 1$ maps the latter isomorphically onto the former.

Proof. By Lemma 3.1(i) both $L^{p}(A) \otimes_{U} \mathbb{Z}$ and $M^{p}(A) \otimes_{U} \mathbb{Z}$ are direct sums of a free abelian group and an elementary abelian $p$-group. Consider the maps in (2.6). By trivializing the $U$-action we obtain homomorphisms

$$
M^{p}(A) \otimes_{U} \mathbb{Z} \stackrel{\theta_{p} \otimes 1}{\longrightarrow} L^{p}(A) \otimes_{U} \mathbb{Z} \stackrel{\eta_{p} \otimes 1}{\longrightarrow} M^{p}(A) \otimes_{U} \mathbb{Z}, \quad \theta_{k} \eta_{k} \otimes 1=(p-2) !
$$

So the restriction of the composite $\theta_{k} \eta_{k} \otimes 1$ to the torsion subgroup of the tensor product $M^{p}(A) \otimes_{U} \mathbb{Z}$, an elementary abelian $p$-group, is multiplication by $(p-2)$ !, that is, it is an isomorphism. It follows that the homomorphism $\theta_{p} \otimes 1$ maps the torsion subgroup of $M^{p}(A) \otimes_{U} \mathbb{Z}$ isomorphically into the torsion subgroup of $L^{p}(A) \otimes_{U} \mathbb{Z}$. To prove the proposition, we need to verify that this map is also surjective, that is, the homomorphism $\theta_{k} \otimes 1$ maps the torsion subgroup of $M^{p}(A) \otimes_{U} \mathbb{Z}$ isomorphically onto the torsion subgroup of $L^{p}(A) \otimes_{U} \mathbb{Z}$. But this is true since otherwise the restriction of $\eta_{p} \otimes 1$ to the torsion subgroup of $L^{p}(A) \otimes_{U} \mathbb{Z}$ would have a non-trivial kernel. This, however, is not the case, as follows from the exactness of (3.1). Since $B^{p}(A) \otimes_{U} \mathbb{Z}$ is free abelian by Lemma 3.2, and $H_{1}\left(M^{p}(A)\right)$ is torsion by Lemma 3.1(ii), there cannot be any torsion elements in the kernel of $\eta_{p} \otimes 1$. This proves the proposition.

In the next section we fill in the gap left in the proof of Lemma 3.2.

\section{The Degree $p$ Lie POWER IN CHARACTERIStic $p$}

In this section $V$ denotes a vector space over a field $K$ of prime characteristic $p$. Moreover, we will assume that $V$ is a module for the polynomial ring $K[X]$ where $X$ is a finite set of indeterminates. Otherwise we will use all the notation introduced in Section 2, in particular, $L^{p}(V), M^{p}(V)$ and $T^{p}(V)$ are the $p$ th Lie, metabelian Lie, and tensor powers of $V$, respectively, $B^{p}(V)=L^{p}(V) \cap L(V)^{\prime \prime}$ is the kernel of the natural projection $L^{p}(V) \rightarrow M^{p}(V)$, and all of these will be regarded as $K[X]$-modules under the derivation action. Recall that $L^{p}(V)$ may be regarded as a submodule of the tensor power $T^{p}(V)$, and hence $B^{p}(V)$ is also a submodule of $T^{p}(V)$.

Lemma 4.1. The submodule $B^{p}(V)$ is a direct summand of the $K[X]$-module $T^{p}(V)$.

This result is essentially proved as Theorem 3.1 in [6], except that there the module $V$ is assumed to be finite-dimensional. In what follows we reproduce the 
proof from [6] with some minor amendments necessary to accommodate infinite dimensional modules.

Proof. For each $r \geq 1$ choose a basis $\mathcal{B}^{(r)}$ of $L^{r}(V)$ and let $\mathcal{B}=\bigcup_{r} \mathcal{B}^{(r)}$. Thus $\mathcal{B}$ is a basis of $L(V)$. For $b \in \mathcal{B}$, let $\operatorname{deg}(b)$ denote the degree of $b$, that is, $\operatorname{deg}(b)=r$ for $b \in \mathcal{B}^{(r)}$. Order $\mathcal{B}$ in any way subject to $b<b^{\prime}$ whenever $\operatorname{deg}(b)<\operatorname{deg}\left(b^{\prime}\right)$. By the Poincaré-Birkhoff-Witt Theorem, $T^{p}(V)$ has a basis $\mathcal{C}$ consisting of all products of the form $b_{1} \otimes b_{2} \otimes \cdots \otimes b_{k}$ with $b_{1}, \ldots, b_{k} \in \mathcal{B}, b_{1} \leq b_{2} \leq \cdots \leq b_{k}$ and $\operatorname{deg}\left(b_{1}\right)+\cdots+\operatorname{deg}\left(b_{k}\right)=p$. More specifically, any basis element $c \in \mathcal{C}$ has the form

$$
c=b_{1}^{(1)} \otimes \cdots \otimes b_{k_{1}}^{(1)} \otimes b_{1}^{(2)} \otimes \cdots \otimes b_{k_{2}}^{(2)} \otimes \cdots \otimes b_{1}^{(p)} \otimes \cdots \otimes b_{k_{p}}^{(p)},
$$

where $k_{1}, \ldots, k_{p}$ are non-negative integers such that $k_{1}+2 k_{2}+\cdots+p k_{p}=p$ and where, for $i=1, \ldots, p$, we have $b_{1}^{(i)}, \ldots, b_{k_{i}}^{(i)} \in \mathcal{B}^{(i)}$ and $b_{1}^{(i)} \leq \cdots \leq b_{k_{i}}^{(i)}$. We call the $p$-tuple $\left(k_{1}, \ldots, k_{p}\right)$ the type of $c$ and denote it by type $(c)$. Let $\Omega$ denote the set of all such types. We order $\Omega$ (lexicographically) by $\left(k_{1}, \ldots, k_{p}\right)>\left(k_{1}^{\prime}, \ldots, k_{p}^{\prime}\right)$ if for some $j \in\{1, \ldots, p\}$ we have $k_{i}=k_{i}^{\prime}$ for all $i<j$ but $k_{j}>k_{j}^{\prime}$. Using this ordering, write $\Omega=\left\{\omega_{1}, \omega_{2}, \ldots, \omega_{m}\right\}$ where $\omega_{1}>\omega_{2}>\cdots>\omega_{m}$. Thus $\omega_{1}=(p, 0, \ldots, 0)$ and $\omega_{m}=(0, \ldots, 0,1)$.

For $i=1, \ldots, m$, define $\mathcal{C}_{i}=\left\{c \in \mathcal{C}: \operatorname{type}(c)=\omega_{i}\right\}$ and let $X_{i}$ denote the subspace of $T^{p}(V)$ spanned by $\mathcal{C}_{i} \cup \mathcal{C}_{i+1} \cup \cdots \cup \mathcal{C}_{m}$. Also, write $X_{m+1}=0$. Thus

$$
T^{p}(V)=X_{1}>X_{2}>\cdots>X_{m}>X_{m+1}=0 .
$$

Note that $X_{i} / X_{i+1}$ has basis $\mathcal{C}_{i}$ modulo $X_{i+1}$. Furthermore, $\mathcal{C}_{1}$ consists of all products $b_{1}^{(1)} \otimes b_{2}^{(1)} \otimes \cdots \otimes b_{p}^{(1)}$ with $b_{1}^{(1)}, \ldots, b_{p}^{(1)} \in \mathcal{B}^{(1)}$ and $b_{1}^{(1)} \leq \cdots \leq b_{p}^{(1)}$. Also, $\mathcal{C}_{m}=\mathcal{B}^{(p)}$ and $X_{m}=L^{p}(V)$.

Let $i \in\{1, \ldots, m\}$ where $\omega_{i}=\left(k_{1}, \ldots, k_{p}\right)$. For $c \in \mathcal{C}_{i}$ written as in (4.1) it is well known and easy to verify that the value of $c$ modulo $X_{i+1}$ is unchanged by any permutation of the factors $b_{1}^{(1)}, \ldots, b_{k_{p}}^{(p)}$. In particular, for all $\pi_{1} \in \operatorname{Sym}\left(k_{1}\right), \ldots$, $\pi_{p} \in \operatorname{Sym}\left(k_{p}\right)$, we have

$$
b_{\pi_{1}(1)}^{(1)} \otimes \cdots \otimes b_{\pi_{1}\left(k_{1}\right)}^{(1)} \otimes \cdots \otimes b_{\pi_{p}(1)}^{(p)} \otimes \cdots \otimes b_{\pi_{p}\left(k_{p}\right)}^{(p)}+X_{i+1}=c+X_{i+1} .
$$

It follows easily that $X_{i}$ is a $K[X]$-submodule of $T^{p}(V)$. For $c$ written as before let $\bar{c} \in S^{k_{1}}\left(L^{1}(V)\right) \otimes \cdots \otimes S^{k_{p}}\left(L^{p}(V)\right)$ be defined by

$$
\bar{c}=\left(b_{1}^{(1)} \circ \cdots \circ b_{k_{1}}^{(1)}\right) \otimes\left(b_{1}^{(2)} \circ \cdots \circ b_{k_{2}}^{(2)}\right) \otimes \cdots \otimes\left(b_{1}^{(p)} \circ \cdots \circ b_{k_{p}}^{(p)}\right) .
$$

Clearly $\left\{\bar{c}: c \in \mathcal{C}_{i}\right\}$ is a basis of $S^{k_{1}}\left(L^{1}(V)\right) \otimes \cdots \otimes S^{k_{p}}\left(L^{p}(V)\right)$. Furthermore, it follows easily from (4.2) that the linear map given by $c+X_{i+1} \mapsto \bar{c}$ is a $K[X]$-module isomorphism from $X_{i} / X_{i+1}$ to $S^{k_{1}}\left(L^{1}(V)\right) \otimes \cdots \otimes S^{k_{p}}\left(L^{p}(V)\right)$. Thus

$$
X_{i} / X_{i+1} \cong S^{k_{1}}\left(L^{1}(V)\right) \otimes \cdots \otimes S^{k_{p}}\left(L^{p}(V)\right) .
$$

Suppose that $i \in\{2, \ldots, m-1\}$ where $\omega_{i}=\left(k_{1}, \ldots, k_{p}\right)$. Thus $k_{1}, \ldots, k_{p}<p$ (and, in fact, $k_{p}=0$ ). Let $\sigma_{i}: S^{k_{1}}\left(L^{1}(V)\right) \otimes \cdots \otimes S^{k_{p}}\left(L^{p}(V)\right) \rightarrow T^{p}(V)$ be the linear map defined on the basis $\left\{\bar{c}: c \in \mathcal{C}_{i}\right\}$ by

$$
\sigma_{i}(\bar{c})=\frac{1}{k_{1} ! \cdots k_{p} !} \sum_{\substack{\pi_{1} \in \operatorname{Sym}\left(k_{1}\right), \ldots, \pi_{p} \in \operatorname{Sym}\left(k_{p}\right)}} b_{\pi_{1}(1)}^{(1)} \otimes \cdots \otimes b_{\pi_{1}\left(k_{1}\right)}^{(1)} \otimes \cdots \otimes b_{\pi_{p}(1)}^{(p)} \otimes \cdots \otimes b_{\pi_{p}\left(k_{p}\right)}^{(p)},
$$


where $c$ is written as in (4.1) It is straightforward to verify that $\sigma_{i}$ is a $K[X]$ module homomorphism. It follows easily from (4.2) that $\sigma_{i}(\bar{c}) \in X_{i}$ and, indeed, $\sigma_{i}(\bar{c})+X_{i+1}=c+X_{i+1}$ for all $c \in \mathcal{C}_{i}$. Thus the map $\sigma_{i}$ is injective and we have $X_{i}=\operatorname{Im}\left(\sigma_{i}\right) \oplus X_{i+1}$. Since $X_{m}=L^{p}(V)$ we therefore have

$$
X_{2}=\operatorname{Im}\left(\sigma_{2}\right) \oplus \cdots \oplus \operatorname{Im}\left(\sigma_{m-1}\right) \oplus L^{p}(V) .
$$

Let $W$ be the submodule of $X_{2}$ given by

$$
W=\operatorname{Im}\left(\sigma_{2}\right) \oplus \cdots \oplus \operatorname{Im}\left(\sigma_{m-1}\right) \oplus\left(L(V)^{\prime \prime} \cap L^{p}(V)\right) .
$$

Consider the maps

$$
\alpha: T^{p}(V) \rightarrow V \otimes V^{p-1}, \quad \beta: V \otimes V^{p-1} \rightarrow T^{p}(V)
$$

defined by

$$
a_{1} \otimes a_{2} \otimes \cdots \otimes a_{p} \mapsto a_{1} \otimes\left(a_{2} \circ \cdots \circ a_{p}\right)
$$

and

$$
a_{1} \otimes\left(a_{2} \circ \cdots \circ a_{p}\right) \mapsto \frac{1}{(p-1) !} \sum_{\pi} a_{1} \otimes a_{\pi(2)} \otimes \cdots \otimes a_{\pi(p)},
$$

where $a_{i} \in V$ and $\pi$ runs over all permutations of $\{2,3, \ldots, c\}$. Then $\alpha$ is surjective and the composite $\beta \alpha$ is the identity map on $V \otimes V^{p-1}$. Hence we have a direct decomposition

$$
T^{p}(V)=\operatorname{Ker}(\alpha) \oplus \operatorname{Im}(\beta) \cong \operatorname{Ker}(\alpha) \oplus\left(V \otimes V^{p-1}\right) .
$$

We claim that $\operatorname{Ker}(\alpha)=W$. It is easily seen that $W$ is contained in $\operatorname{Ker}(\alpha)$. To verify that we have actually equality, note that the elements

$$
b_{1}^{(1)} \otimes b_{2}^{(1)} \otimes \cdots \otimes b_{p}^{(1)} \quad \text { with } b_{1}^{(1)}, \ldots, b_{p}^{(1)} \in \mathcal{B}^{(1)} \text { and } b_{1}^{(1)} \leq \cdots \leq b_{p}^{(1)}
$$

form a basis of $T^{p}(V)$ modulo $X_{2}$. Furthermore, the Lie products

$$
\left[b_{1}^{(1)}, b_{2}^{(1)}, \ldots, b_{p}^{(1)}\right] \quad \text { with } b_{1}^{(1)}, \ldots, b_{p}^{(1)} \in \mathcal{B}^{(1)} \text { and } b_{1}^{(1)}>b_{2}^{(1)} \leq \cdots \leq b_{p}^{(1)}
$$

form a basis of $L^{p}(V)$ modulo $L^{p}(V) \cap L(V)^{\prime \prime}$ (see Section 2). It follows that the elements (4.5) together with the elements (4.6) form a basis of $T^{c}(V)$ modulo $W$. Moreover, the images of these elements under the map $\alpha$ form a basis of $V \otimes V^{p-1}$. Indeed, we have

$$
\left(b_{1}^{(1)} \otimes b_{2}^{(1)} \otimes \cdots \otimes b_{p}^{(1)}\right) \alpha=b_{1}^{(1)} \otimes\left(b_{2}^{(1)} \circ \cdots \circ b_{p}^{(1)}\right)
$$

and

$$
\left(\left[b_{1}^{(1)}, b_{2}^{(1)}, \ldots, b_{p}^{(1)}\right]\right) \alpha=b_{1}^{(1)} \otimes\left(b_{2}^{(1)} \circ \cdots \circ b_{p}^{(1)}\right)-b_{2}^{(1)} \otimes\left(b_{1}^{(1)} \circ \cdots \circ b_{p}^{(1)}\right) .
$$

This can easily be seen from the short exact sequence (2.3) (with $V$ instead of $A$ ). Indeed, the elements (4.7) are mapped by $\kappa_{p}$ one-to-one onto the canonical basis of $V^{p}$, and the elements (4.8) are precisely the images of the canonical basis elements of $M^{p}(V)$ under the map $\mu_{p}$. Consequently, we have the desired equality $\operatorname{Ker}(\alpha)=W$, and so $T^{p}(V)=W \oplus \operatorname{Im}(\beta)$. By (4.4), $L^{p}(V) \cap L(V)^{\prime \prime}$ is a direct summand of $W$. Thus $L^{p}(V) \cap L(V)^{\prime \prime}$ is a direct summand of $T^{p}(V)$ and we have Lemma 4.1.

Now the result we need to complete the proof of Proposition 3.3 follows easily. 
Corollary 4.2. If $V$ is a free $K[X]$-module, then $B^{p}(V)$ is a projective $K[X]$ module.

Proof. If $V$ is a free $K[X]$-module, then the tensor power $T^{p}(V)$ is also a free $K[X]$ module, see [14, Lemma 5.2]). Since $B^{p}(V)$ is a direct summand of $T^{p}(V)$, it is projective.

\section{THE MAIN RESULT}

In this Section $L$ denotes a free Lie ring of finite rank with free generating set $X$. Our aim is to determine the torsion subgroup of the additive group of the quotient (1.1). In view of the short exact sequence

$$
0 \rightarrow\left(L^{\prime}\right)^{c} /\left[\left(L^{\prime}\right)^{c}, L\right] \rightarrow L /\left[\left(L^{\prime}\right)^{c}, L\right] \rightarrow L /\left(L^{\prime}\right)^{c} \rightarrow 0,
$$

this is a free central extension of the free (nilpotent-of-class-c-1)-by-abelian Lie ring $L /\left(L^{\prime}\right)^{c}$. The additive structure of the latter is well-understood. Its underlying abelian group is free abelian [3]. Consequently, any torsion elements must be contained in the central quotient $\left(L^{\prime}\right)^{c} /\left[\left(L^{\prime}\right)^{c}, L\right]$, and it is this quotient we will focus on from now on. By the Shirshov-Witt Theorem, the derived ideal $L^{\prime}$ is itself a free Lie ring, namely, the free Lie ring on $L^{\prime} / L^{\prime \prime}: L^{\prime}=L\left(L^{\prime} / L^{\prime \prime}\right)$. This is a graded Lie ring and its degree $c$ homogeneous component $L^{c}\left(L^{\prime} / L^{\prime \prime}\right)$ is isomorphic to the lower central quotient

$$
L^{c}\left(L^{\prime} / L^{\prime \prime}\right) \cong\left(L^{\prime}\right)^{c} /\left(L^{\prime}\right)^{c+1} .
$$

The adjoint representation induces on these lower central quotients the structure of an $L / L^{\prime}$-module, and hence of a module for its universal envelope $U=U\left(L / L^{\prime}\right)$. The latter may be identified with the polynomial ring on $X: U=\mathbb{Z}[X]$. Thus (5.1) is actually a $U$-module isomorphism. In view of the canonical isomorphism

$$
\left(\left(L^{\prime}\right)^{c} /\left(L^{\prime}\right)^{c+1} \otimes_{U} \mathbb{Z} \cong\left(L^{\prime}\right)^{c} /\left[\left(L^{\prime}\right)^{c}, L\right],\right.
$$

trivializing the $U$-action on both sides of (4.1) gives an isomorphism

$$
\left(L^{\prime}\right)^{c} /\left[\left(L^{\prime}\right)^{c}, L\right] \cong L^{c}\left(L^{\prime} / L^{\prime \prime}\right) \otimes_{U} \mathbb{Z} .
$$

We will exploit this isomorphism to investigate the additive structure of the quotient on the left hand side by examining the tensor product on the right hand side.

Suppose that $L$ has rank 2 and, say, $X=\{x, y\}$. Then $L^{\prime} / L^{\prime \prime}$ is a free cyclic module over the polynomial $\operatorname{ring} U=\mathbb{Z}[x, y]$ with free generator $[y, x]$, see $[1$, Proof of Theorem 6.1]. If $c$ is a prime, say $c=p$, then Proposition 3.3 applies to the tensor product on the right hand side of (5.2). Hence this tensor product is a direct sum of a free abelian group and an elementary abelian $p$-group. Moreover, the torsion subgroup is the image in $L^{p}\left(L^{\prime} / L^{\prime \prime}\right) \otimes_{U} \mathbb{Z}$ of the torsion subgroup of $M^{p}\left(L^{\prime} / L^{\prime \prime}\right) \otimes_{U} \mathbb{Z}$ under the map $\theta_{p} \otimes 1$. A complete description of the latter is given in [1, Corollary 6.2]. The elements

$$
[[u, y],[u, x], \underbrace{u, \ldots, u}_{p-2}] \otimes 1
$$


where $u=[y, x, \underbrace{x, \ldots, x}_{s}, \underbrace{y, \ldots, y}_{t}]$ with $s, t \geqslant 0$ form a basis of this torsion subgroup as a $\mathbb{Z}_{p}$-module. Applying $\theta_{p} \otimes 1$ to such a basis element gives

$$
\frac{(p-2) !}{p} \sum_{i=0}^{p-1}([[u, y], \underbrace{u, \ldots, u}_{i},[u, x], \underbrace{u, \ldots, u}_{p-2-i}]-[[u, x], \underbrace{u, \ldots, u}_{i},[u, y], \underbrace{u, \ldots, u}_{p-2-i}]) \otimes 1 .
$$

Since this is an element of order $p$ we can drop the factor of $(p-2)$ ! in the statement of our main result, which summarizes the discussion in this final section.

Theorem 5.1. Let $L$ be a free Lie ring of rank 2 with free generators $x$ and $y$, and let $p$ be a prime. Then the quotient $\left(L^{\prime}\right)^{p} /\left[\left(L^{\prime}\right)^{p}, L\right]$ is a direct sum of a free abelian group and an elementary abelian p-group. Modulo $\left[\left(L^{\prime}\right)^{p}, L\right]$ the elements

$$
\frac{1}{p} \sum_{i=0}^{p-1}([[u, y], \underbrace{u, \ldots, u}_{i},[u, x], \underbrace{u, \ldots, u}_{p-2-i}]-[[u, x], \underbrace{u, \ldots, u}_{i},[u, y], \underbrace{u, \ldots, u}_{p-2-i}])
$$

where $u=[y, x, \underbrace{x, \ldots, x}_{s}, \underbrace{y, \ldots, y}_{t}]$ with $s, t \geqslant 0$ form a basis of this torsion subgroup as a $\mathbb{Z}_{p}$-module.

The legality of the factor $1 / p$ in the statement of the theorem is explained in Section 2.

\section{REFERENCES}

[1] Maria Alexandrou and Ralph Stöhr, Free centre-by-nilpotent-by-abelian Lie rings of rank 2, J. Austral. Math. Soc. 102 (2017), no.1, 63-73.

[2] Yu. A. Bakhturin, Identical relations in Lie algebras, Nauka, Moscow, 1985 (Russian). English translation: VNU Science Press, Utrecht, 1987.

[3] L.A. Bokut', A basis of free polynilpotent Lie algebras (Russian), Algebra i Logika 2 (1963), no. $4,13-18$.

[4] R.M.Bryant, L.G. Kovács and Ralph Stöhr, Lie powers of modules for groups of prime order, Proc. London Math. Soc. 84 (2002), 334-374.

[5] R.M.Bryant and Ralph Stöhr, On the module structure of free Lie algebras, Trans. Amer. Math. Soc. 352 (2000), 901-934.

[6] R.M.Bryant and Ralph Stöhr, Lie powers in prime degree, Q. J. Math. 56 (2005), 473-489.

[7] Vesselin Drensky, Torsion in the additive group of relatively free Lie rings, Bull. Austral. Math. Soc. 33 (1986), no. 1, 81-87.

[8] Chander Kanta Gupta, The free centre-by-metabelian groups, J. Austral. Math. Soc. 16 (1973), 294-299.

[9] T. Hannebauer and R. Stöhr, Homology of groups with coefficients in free metabelian Lie powers and exterior powers of relation modules and applications to group theory, in Proc. Second Internat. Group Theory Conf., Bressanone, 1989, Rend. Circ. Mat. Palermo (2) Suppl. 23 (1990), 77-113.

[10] Marianne Johnson and Ralph Stöhr, Free central extensions of groups and modular Lie powers of relation modules, Proc. Amer. Math. Soc., 138 (2010), no. 11, 3807-3814.

[11] L.G. Kovács and Ralph Stöhr, Free centre-by-metabelian Lie algebras in characteristic 2, Bull.Lond. Math. Soc., 46 (2014), 491-502.

[12] Yu. V. Kuz'min, Free center-by-metabelian groups, Lie algebras and $\mathcal{D}$-groups (Russian), Izv. Akad. Nauk SSSR Ser. Mat. 41 (1977), no. 1, 3-33, 231. English translation: Math. USSR Izvestija 11 (1977), no. 1, 1-30. 
[13] Magnus, W., Karras, A, Solitar, D. Combinatorial Group Theory, Wiley-Interscience, New York, 1966.

[14] Nil Mansuroğlu and Ralph Stöhr, Free centre-by-metabelian Lie rings, Q. J. Math. 65 (2014), no. $2,555-579$.

[15] Ralph Stöhr, On torsion in free central extensions of some torsion-free groups, J. Pure Appl. Algebra 46 (1987), no. 2-3, 249-289.

[16] Ralph Stöhr, Homology of free Lie powers and torsion in groups, Israel J. Math. 84 (1993), 65-87.

School of Mathematics, University of Manchester, Alan Turing Bullding, Manchester, M13 9PL, United Kingdom

E-mail address: Ralph.Stohr@manchester.ac.uk 